Dublin. Dr Vincent Agyapong is a senior registrar and lecturer in psychiatry, St Patrick's Hospital and University of Dublin (Trinity College) Dublin. Leah Quinlivan is a PhD Research Fellow, Department of Psychiatry \& Mental Health Research, School of Medicine \& Medical Sciences, University College Dublin. Dr John Tobin is a consultant psychiatrist, St Bricin's Hospital, Dublin. Professor Kevin Malone is head of the Department of Psychiatry \& Mental Health Research, School of Medicine $\&$ Medical Sciences, University College Dublin, Ireland.

\section{References}

1 Giorgi-Guarnieri D, Norko MA. Stalking: introduction, definition and epidemiology. In Stalking - Psychiatric Perspectives and Practical Approaches (ed. DA Pinals): 3-6. Oxford University Press, 2007.

2 Meloy JR. The Psychology of Stalking: Clinical and Legal Perspectives. Academic Press, 1998.

3 Westrup D, Fremouw WJ. Stalking behaviour: a literature review and suggested functional analytic assessment technology. Aggress Violent Behav 1998; 3: 255-74

4 Mullen PE, Pathe M, Purcell R. Stalkers and their Victims (2nd edn). Cambridge University Press, 2008.

5 Romans JS, Hays JR, White TK. Stalking and related behaviours experienced by counselling centre staff members from current or former clients. Prof Psychol 1996; 27: 595-9.
6 Sandberg DA, McNeil DE, Binder RL. Stalking, threatening and harassing and harassing behaviour by psychiatric patients towards clinicians. J Am Acad Psychiatry Law 2002; 30: 221-9.

7 Sandberg DA, McNeil DA, Binder RL. Characteristics of psychiatric inpatients who stalk, threaten, or harass hospital staff after discharge. Am J Psychiatry 1998; 155: 1102-5.

8 Krammer A, Stepan A, Baranyi A, Kapfhammer HP, Rothenhäusler HB. The effects of stalking on psychiatrists, psychotherapists and psychologists. Prevalence of stalking and its emotional impact [in German]. Nervenarzt 2007; 78: 809-17.

9 Galeazzi GM, Elkins BA, Curci P. Emergency psychiatry: the stalking of mental health professionals by patients. Psychiatr Serv 2005; 56: 137-8.

10 Royal College of Psychiatrists. 1 in 10 psychiatrists are victims of stalking: 1 in 3 report harassment from stalking behaviours. Roya College of Psychiatrists, 2008 (http://www.rcpsych.ac.uk/press/ pressreleases2008/pr985.aspx).

11 Mclvor RJ, Potter L, Davies L. Stalking behaviour by patients towards psychiatrists in a large mental health organisation. Int J Soc Psychiatry 2008; 54: 350-7.

12 Ashmore R, Jones J, Jackson A, Smoyak S. A survey of mental health nurses experiencing stalking. J Psychiatr Ment Health Nurs 2006; 13 562-9.

13 Mullen PE, Pathe M, Purcell R, Stuart GW. Study of stalkers. Am J Psychiatry 1999; 156: 1244-9.

\title{
Assessing whether psychiatric trainees feel safe in the workplace
}

\author{
Catia Acosta, ${ }^{1}$ James Warner, ${ }^{2}$ Michael Kopelman, ${ }^{1}$ Ramin Nilforooshan ${ }^{3}$
}

The Psychiatrist (2012), 36, 93-96, doi: 10.1192/pb.bp.110.032987

${ }^{1}$ South London and Maudsley NHS Foundation Trust; ${ }^{2}$ Central and North West London NHS Foundation Trust; ${ }^{3}$ Surrey and Borders Partnership NHS Foundation Trust

Correspondence to Ramin

Nilforooshan

(ramin.nilforooshan@nhs.net)

First received 7 Oct 2010, final revision 24 Jul 2011, accepted 21 Sep 2011

\begin{abstract}
Aims and method Previous studies have shown that 17 to $60 \%$ of psychiatric trainees have been physically or verbally assaulted. To measure the frequency of assaults and the trainees' reactions, we conducted a retrospective self-reported survey of attendees at MRCPsych teaching courses in south London and at an annual meeting of psychiatric trainees.

Results Overall, $64 \%$ of the questionnaires distributed were returned completed. Of the trainees who responded, $41 \%$ had been physically assaulted at least once and $89 \%$ had been verbally assaulted. As a result of the assault, $34 \%$ of trainees were subsequently more risk aware and $11 \%$ were now hesitant to assess patients with a history of violence. There was no association between the level of training or attendance at a breakaway training course and having been subject to physical assault.
\end{abstract}

Clinical implications Our study showed unacceptable levels of physical and verbal assault on psychiatric trainees and an important effect of those incidents on clinical practice.

Declaration of interest None.
Previous studies have shown that many psychiatrists have been assaulted and/or threatened while at work, with prevalence ranging from 17 to $60 \% .^{1-5}$ Few studies on this subject have been undertaken in England. Davies noted that over the year, $17 \%$ of respondents reported one or more assaults (of those, $42 \%$ were assaulted more than once) and $32 \%$ reported one or more threats. ${ }^{1}$ Most of the assaults $(61 \%)$ were committed by patients in general adult 
psychiatry settings; half occurred during urgent assessments; and junior doctors were more likely to have experienced an incident, independently of gender., ${ }^{1,2}$

\section{Junior doctors' experiences of assault at work - research evidence}

It has been suggested that the risk of threat and assault decreases with seniority, mostly because of experience than assignment to different duties, suggesting that training in management of violence and closer supervision earlier in training might have a role in modifying risk. ${ }^{1}$ Generally, junior doctors feel less safe at work than consultants, probably because of less experience in risk assessment, high exposure to new patients and out-of-hours services, and difficulties in accessing colleagues to carry out joint assessments. ${ }^{6}$ Almost $50 \%$ frequently feel vulnerable or fearful for personal safety at work, although those working in the community felt less concern for their safety. ${ }^{2}$

In a survey by Dibben et $a l,{ }^{6} 55 \%$ of senior house officers felt sufficiently threatened by a patient to terminate the interview. Also, $64 \%$ of specialist registrars and $52 \%$ of consultants reported feeling threatened by patients or their relatives. Other studies revealed that less than $20 \%$ of incidents were reported to line managers but $78 \%$ were documented in the patient's case notes. ${ }^{1,3}$ Dibben et $a l^{6}$ compared incident reporting by the three groups of doctors and found that $33 \%$ of senior house officers reported incidents compared with only $18 \%$ of specialist registrars and $11 \%$ of consultants. One of the reasons for not reporting incidents was that violence was seen as 'part of the job'? Other reasons were feeling that their reports would not be taken seriously, and in almost $73 \%$ of cases, doctors were not aware of guidelines for reporting incidents. ${ }^{3}$

Davies $^{1}$ found that only $10 \%$ of those assaulted took time away from clinical duties, but the psychological consequences of the violence suffered were not assessed. In a study by Kidd $\&$ Stark, ${ }^{3}$ doctors were asked to indicate whether support or counselling had been offered after each incident. Only $4 \%$ were offered support after one incident, and $22 \%$ said it was unnecessary.

Most of the studies published to date were very comprehensive regarding the risk and degree of assault and threat experienced by doctors working in psychiatry. We focused on trainees as they seem to be the more vulnerable and at risk. Moreover, none of the studies assessed the potential physical and/or psychological consequences or the subsequent changes in clinical practice that these violent episodes generate. This is something we have examined in our study, carried out in a mental health trust in south London.

\section{Method}

We developed a questionnaire informed by the previous research on this issue. ${ }^{1-4}$ This questionnaire was devised to measure the frequency of physical and/or verbal assault on psychiatric trainees at work. We also asked about the physical and psychological consequences of assaults and what, if any, changes they had on the way respondents undertook their clinical duties. The questionnaire was
Table 1 Frequency of physical and verbal assaults on trainees

\begin{tabular}{lccc} 
& \multicolumn{3}{c}{$n(\%)$} \\
\cline { 2 - 4 } Demographics & Male & Female & Total \\
\hline Total & $60(55.6)$ & $48(44.4)$ & $108(100)$ \\
\hline Age, years & $18(30.0)$ & $19(39.6)$ & $37(34.3)$ \\
$\quad<30$ & $39(65.0)$ & $28(58.3)$ & $67(62.2)$ \\
$30-39$ & $3(5.0)$ & $1(2.1)$ & $4(3.7)$ \\
$\geqslant 40$ & & & \\
\hline Level of training & $42(70.0)$ & $34(70.8)$ & $76(70.4)$ \\
CT/ST1-3 & $18(30.0)$ & $14(29.2)$ & $32(29.6)$ \\
$\quad$ SpR/ST4-6 & & & \\
\hline Attended awareness & & & \\
disengagement skills & $43(71.7)$ & $28(58.3)$ & $71(65.7)$ \\
training & $29(48.3)$ & $15(31.3)$ & $44(40.7)$ \\
\hline Assault & $55(91.7)$ & $41(85.4)$ & $96(88.9)$ \\
$\quad \begin{array}{l}\text { Physical } \\
\text { Verbal }\end{array}$ &
\end{tabular}

$\mathrm{CT}$, core trainee; $\mathrm{SpR}$, specialist registrars; $\mathrm{ST}$, specialist trainee.

distributed to trainees who attended an MRCPsych teaching day in south London and to attendees at the second annual meeting of psychiatric trainees at the London Deanery (attendees at this meeting where asked not to complete the questionnaire if they had already done so).

The questionnaire was anonymous, with a mixture of 'Yes'/'No' and multiple choice questions and free text. There was space at the end for comments. We used Stata version 10 for Windows to analyse any relationship between physical assault and the trainee's level of training or attendance at a breakaway training course in the previous year.

\section{Results}

Of the 170 questionnaires distributed, 108 were completed $(64 \%) ; 76(70 \%)$ were at the core trainee (CT)/specialist trainee (ST) 1-3 level of training and the remaining 32 (30\%) were specialist registrars or at the ST4-6 level. The sample is described in Table 1 . We divided the trainees into junior and senior trainees and into three age groups. The age cohorts were chosen to reflect differing levels of maturity and different training epochs.

Table 2 Change in clinical practice of trainees after having been physically or verbally assaulted

\begin{tabular}{lrr} 
Change & $\%$ & $n$ \\
\hline More aware of risks & 34.3 & 37 \\
\hline More aware of the environment during interview & 29.6 & 32 \\
\hline Hesitant on assessing patients with a history & 11.1 & 12 \\
\hline of violence & 9.3 & 10 \\
\hline Uncomfortable at work & 6.5 & 7 \\
\hline Uncomfortable when alone with patients & 25.0 & 27 \\
\hline No answer & 5.6 & 6 \\
\hline
\end{tabular}


Of the 44 trainees who had been physically assaulted, $30(68 \%)$ had attended a breakaway training course in the previous 12 months and $14(32 \%)$ had never attended such a course. Of trainees who were not physically assaulted $(n=64), 41(64 \%)$ had attended a breakaway course. There was no statistically significant relationship between having been physically assaulted and the trainee's level of training $\left(\chi^{2}=3.325\right.$, d.f. $\left.=4, P=0.505\right)$; or between being assaulted and having attended a breakaway course $\left(\chi^{2}=0.196\right.$, $P=0.658$ ). The relatively small sample size in each age group did not permit further analyses. Of the trainees that had been physically assaulted, $70 \%$ were alone with the patient at the time of the incident. We did not question whether doctors carried a personal alarm when interviewing a patient or whether they had a chance to use it.

\section{Incident reporting and support received}

Forty trainees (41\%) who were physically or verbally assaulted had not reported the incident. Of those who did, $92(66 \%)$ reported them only in the patient's medical records, 22 (16\%) filled in an incident report form, and 26 (19\%) did both. Physical assault was the type of event most frequently reported on an incident form, or on both the incident form and the patient's medical records. Reasons given for not formally reporting incidents included:

a the physical assault had been minor with no major physical injury

b being assaulted was considered an 'occupational hazard'

c the patient apologised when mentally recovered.

Seven respondents said that verbal assaults were too frequent to report and were considered 'part of the job'; and in three cases, assaults were not reported because the person did not know how to do so. Of those who reported incidents, 59 (43\%) were CT/ST1-3 and 23 (18\%) were senior trainees.

Of the 44 trainees who had been physically assaulted, 2 (2\%) required medical treatment and $4(9 \%)$ took time off work, with 1 person taking half a day off and the remaining 3 a full day.

Only 9 trainees (9\%) of all those who experienced physical and verbal incidents were given post-incident support. In the comments section of the questionnaire, three doctors admitted that they were not even aware that post-incident support was available in the trust; one had to seek support himself as the attitude shown by his supervising consultant was that 'it is part of the job'. Another doctor commented that he felt there should be a clear protocol for reporting violence in the workplace and that this should be given to all staff.

The final question asked the trainees whether an assault, either physical or verbal, had changed the way that they performed their clinical duties. Thirty-seven trainees (34\%) denied that their practice had changed at all after being a victim of an assault (Table 2).

\section{Discussion}

The high response rate to our survey suggests that safety at work is a salient topic for psychiatric trainees and that it merits more awareness by trainees, clinical supervisors, trusts and the Royal College of Psychiatrists. Our results show that nearly all respondents had experienced a verbal assault and $44(41 \%)$ had been physically assaulted while undertaking their clinical duties. This is comparable with findings in other published studies. ${ }^{1-5}$ Rates were similar between junior and senior trainees.

Despite attendance at a breakaway training course being mandatory for all trainees, only 71 (65\%) had participated in such a course. Trainees who had attended breakaway training were no less likely to be subject to physical assault. Breakaway training is not designed to prevent physical assault but rather to minimise the impact of it. Our findings raise the question of whether such courses should continue in the present form or whether a modified version, with more training about how to avoid or better manage potentially risky situations and advice on what to do following an assault, would be more appropriate for junior doctors.

The majority of the trainees reported the incidents only in the patients' medical records, which left trusts unaware of the levels of assaults experienced by their staff. We did not ask specifically in our questionnaire whether doctors had been given training in incident reporting and consequently we cannot comment whether this might have had an influence on the low rate of incident reports.

In keeping with other studies, trainees reported that they were not aware of incident forms or how to complete them. ${ }^{1,3,7}$ Another reason for non-reporting, mostly in relation with verbal abuse, was that it occurs too frequently to report. Given the incidence of verbal abuse and threat identified in this study, we find this alarming. It is also very concerning that assaults and threats are perceived by a significant number of trainees as 'part of the job', something which would be seen as unacceptable in most other professions. Formal training in incident reporting would help improve the situation.

After experiencing an assault the major consequences for trainees were their increased awareness of potential risks, awareness of the environment during a clinical interview, being hesitant to assess a patient with a history of violence, and feeling generally less comfortable at work. Although the first two of these outcomes are desirable, it is regrettable that these potentially protective measures were taken only after having been assaulted. We speculate that the frequency of assaults may affect such trainees' assessment of patients with a history of violence and this may be something which should be investigated.

It is important to protect psychiatric trainees from violence at work. Making the workplace safer, changing the culture to recognise that violence and threats are an occupational hazard and offering more useful training in how to deal with risk situations are priorities. If assessing and managing risk were a part of clinical training, no one should tolerate working in an unsafe environment.

We believe a national survey on this subject is needed, involving psychiatrists in training and non-training posts. We suggest that the perceived risk of aggression may be a 
contributory reason for the recruitment crisis in psychiatry. $^{8,9}$ It is possible that, if this problem were tackled, the recruitment levels might improve, as well as the overall enjoyment by trainees in the work they undertake.

\section{About the authors}

Catia Acosta, CT3 in psychiatry, South London and Maudsley NHS Foundation Trust; James Warner, Consultant and Reader in Older Adults Psychiatry, Central and North West London NHS Foundation Trust; Michael Kopelman, Consultant Neuropsychiatrist and Senior Lecturer, South London and Maudsley NHS Foundation Trust; Ramin Nilforooshan, Consultant of Older Adult Psychiatry, Surrey and Borders Partnership NHS Foundation Trust.

\section{References}

1 Davies S. Assaults and threats on psychiatrists. Psychiatr Bull 2001; 25 $89-91$.
2 Chubb H. Safety awareness among junior psychiatrists and provisions for their safety in the workplace. Psychiatr Bull 1997; 21: 80-3.

3 Kidd B, Stark CR. Violence and junior doctors working in psychiatry. Psychiatr Bull 1992; 16: 144-5.

4 Fottrell E. A study of violent behaviour among patients in psychiatric hospitals. Br J Psychiatry 1980; 136: 216-21.

5 Health Services Advisory Committee. Violence and Aggression to Staff in Health Services. Guidance on Assessment and Management. HSE Books, 1997.

6 Dibben C, O'Shea R, Chang R, Woodger J. Safety for psychiatrists - from trainee to consultant. Psychiatr Bull 2008; 32: 85-7.

7 Lyon JR, Snyder WR, Merrill GL. Under reporting of assaults on staff in a state hospital. Hosp Community Psychiatry 1981; 32: 497-8.

8 Brown TM, Addie K, Eagles JM. Recruitment into psychiatry: views of consultants in Scotland. Psychiatr Bull 2007; 31: 411-3.

9 Brockington IF, Mumford DB. Recruitment into psychiatry. Br J Psychiatry 2002; 180: 307-12. 